\title{
SEARCHES FOR POINT-LIKE AND EXTENDED NEUTRINO SOURCES CLOSE TO THE GALACTIC CENTER USING THE ANTARES NEUTRINO TELESCOPE
}

\section{S. Adrí́n-Martínez ${ }^{1}$, A. Albert ${ }^{2}$, M. André ${ }^{3}$, M. Anghinolfir ${ }^{4}$, G. Anton ${ }^{5}$, M. Ardid ${ }^{1}$, J.-J. Aubert ${ }^{6}$, B. Baret ${ }^{7}$, J. Barrios-Martí ${ }^{8}$, S. Basa ${ }^{9}$, V. Bertin ${ }^{6}$, S. Biagi ${ }^{10,11}$, C. BogazzI ${ }^{12}$, R. Bormuth ${ }^{12,13}$, M. Bou-Cabo ${ }^{1}$, M. C. Bouwhuis ${ }^{12}$,

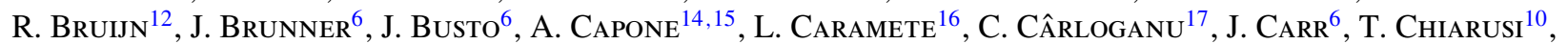

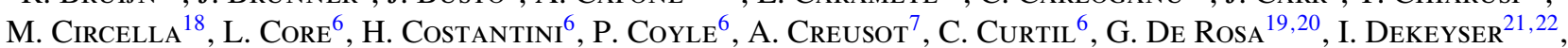

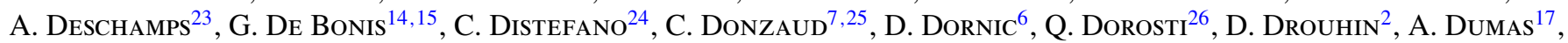

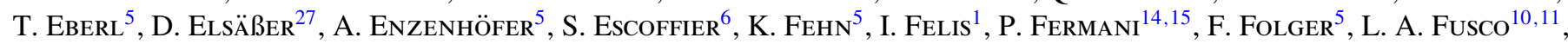
S. Galatì ${ }^{7}$, P. Gay ${ }^{17}$, S. Geißelsöder ${ }^{5}$, K. Geyer ${ }^{5}$, V. Giordano ${ }^{28}$, A. GleiXner ${ }^{5}$, J. P. Gómez-González ${ }^{8}$, K. GraF ${ }^{5}$, G. Guillard ${ }^{17}$, H. van Haren ${ }^{29}$, A. J. Heijboer ${ }^{12}$, Y. Hello $^{23}$, J. J. HernándeZ-Rey ${ }^{8}$, B. Herold ${ }^{5}$, A. Herrero ${ }^{1}$, J. Hößs ${ }^{5}$,

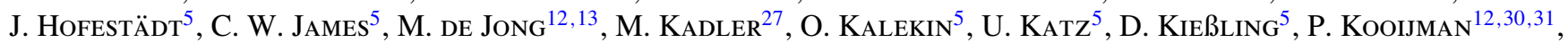

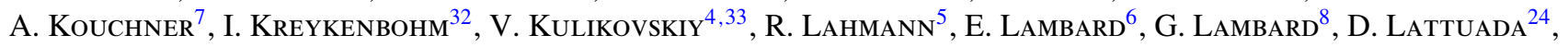

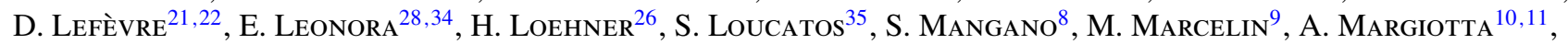
J. A. Martínez-Mora ${ }^{1}$, S. Martini ${ }^{21,22}$, A. Mathieu ${ }^{6}$, T. Michael ${ }^{12}$, P. Migliozzi $^{19}$, C. Mueller $^{32}$, M. NefF $^{5}$, E. Nezri ${ }^{9}$, D. Palioselitis ${ }^{12}$, G. E. Păvălaş ${ }^{16}$, C. Perrina ${ }^{14,15}$, P. Piattellit ${ }^{24}$, V. Popa ${ }^{16}$, T. Pradier $^{36}$, C. Racca $^{2}$, G. Riccobene $^{24}$, R. Richter ${ }^{5}$, K. Roensch ${ }^{5}$, A. Rostovtsev ${ }^{37}$, M. SAldaña ${ }^{1}$, D. F. E. SAmtleben ${ }^{12,13}$, A. SÁnChez-Losa ${ }^{8}$, M. SAnguineti ${ }^{4,38}$, P. Sapienza ${ }^{24}$, J. Schmid ${ }^{5}$, J. Schnabel ${ }^{5}$, S. Schulte ${ }^{12}$, F. Schüßler ${ }^{35}$, T. Seitz ${ }^{5}$, C. Sieger ${ }^{5}$, A. Spies ${ }^{5}$, M. Spurio ${ }^{10,11}$, J. J. M. SteiJger ${ }^{12}$, Th. StolarczyK ${ }^{35}$, M. Taiuti ${ }^{4,38}$, C. Tamburini ${ }^{21,22}$, Y. Tayalati ${ }^{39}$, A. Trovato ${ }^{24}$, B. Vallage ${ }^{35}$, C. ValléE ${ }^{6}$, V. VAn ElewycK ${ }^{7}$, E. Visser ${ }^{12}$, D. Vivolo ${ }^{19,20}$, S. Wagner ${ }^{5}$, J. Wilms ${ }^{32}$, E. De Wolf ${ }^{12,31}$, K. YatKin $^{6}$, H. YePES ${ }^{8}$, J. D. ZORNOZA ${ }^{8}$, AND J. ZÚÑIGA ${ }^{8}$

${ }^{1}$ Institut d'Investigació per a la Gestió Integrada de les Zones Costaneres (IGIC) - Universitat Politècnica de València. C/ Paranimf 1, E-46730 Gandia, Spain ${ }^{2}$ GRPHE - Institut universitaire de technologie de Colmar, 34 rue du Grillenbreit BP 50568, F-68008 Colmar, France

${ }^{3}$ Technical University of Catalonia, Laboratory of Applied Bioacoustics, Rambla Exposició, E-08800 Vilanova i la Geltrú, Barcelona, Spain ${ }^{4}$ INFN - Sezione di Genova, Via Dodecaneso 33, I-16146 Genova, Italy

${ }^{5}$ Friedrich-Alexander-Universität Erlangen-Nürnberg, Erlangen Centre for Astroparticle Physics, Erwin-Rommel-Str. 1, D-91058 Erlangen, Germany ${ }^{6}$ CPPM, Aix-Marseille Université, CNRS/IN2P3, Marseille, France

${ }^{7}$ APC, Université Paris Diderot, CNRS/IN2P3, CEA/IRFU, Observatoire de Paris, Sorbonne Paris Cité, F-75205 Paris, France

${ }^{8}$ IFIC - Instituto de Física Corpuscular, Edificios Investigación de Paterna, CSIC - Universitat de València, Apdo. de Correos 22085 , E-46071 Valencia, Spain

${ }^{9}$ LAM - Laboratoire d'Astrophysique de Marseille, Pôle de l'Étoile Site de Château-Gombert, rue Frédéric Joliot-Curie 38, F-13388 Marseille Cedex 13, France ${ }^{10}$ INFN - Sezione di Bologna, Viale Berti-Pichat 6/2, I-40127 Bologna, Italy

${ }^{11}$ Dipartimento di Fisica dell'Università, Viale Berti Pichat 6/2, I-40127 Bologna, Italy

${ }^{12}$ Nikhef, Science Park, Amsterdam, The Netherlands

${ }^{13}$ Huygens-Kamerlingh Onnes Laboratorium, Universiteit Leiden, The Netherlands

${ }^{14}$ INFN -Sezione di Roma, P.le Aldo Moro 2, I-00185 Roma, Italy

${ }^{15}$ Dipartimento di Fisica dell'Università La Sapienza, P.le Aldo Moro 2, I-00185 Roma, Italy

${ }^{16}$ Institute for Space Sciences, R-77125 Bucharest, Măgurele, Romania

${ }^{17}$ Laboratoire de Physique Corpusculaire, Clermont Univertsité, Université Blaise Pascal, CNRS/IN2P3, BP 10448, F-63000 Clermont-Ferrand, France

${ }^{18}$ INFN - Sezione di Bari, Via E. Orabona 4, I-70126 Bari, Italy

${ }^{19}$ INFN -Sezione di Napoli, Via Cintia, I-80126 Napoli, Italy

${ }^{20}$ Dipartimento di Fisica dell'Università Federico II di Napoli, Via Cintia, I-80126, Napoli, Italy

${ }^{21}$ Aix-Marseille University, CNRS/INSU, IRD, Mediterranean Institute of Oceanography (MIO), UM 110, Marseille, France

22 Université de Toulon, CNRS/INSU, IRD, Mediterrranean Institute of Oceanography (MIO), UM 110, La Garde Cedex, France

${ }^{23}$ Géoazur, Université Nice Sophia-Antipolis, CNRS/INSU, IRD, Observatoire de la Côte d'Azur, Sophia Antipolis, France ${ }^{24}$ INFN - Laboratori Nazionali del Sud (LNS), Via S. Sofia 62, I-95123 Catania, Italy

${ }^{25}$ Univ. Paris-Sud, F-91405 Orsay Cedex, France

${ }^{26}$ Kernfysisch Versneller Instituut (KVI), University of Groningen, Zernikelaan 25, 9747 AA Groningen, The Netherlands

${ }^{27}$ Institut für Theoretische Physik und Astrophysik, Universität Würzburg, Emil-Fischer Strasse 31, D-97074 Würzburg, Germany

${ }^{28}$ INFN - Sezione di Catania, Viale Andrea Doria 6, I-95125 Catania, Italy

${ }^{29}$ Royal Netherlands Institute for Sea Research (NIOZ), Landsdiep 4,1797 SZ 't Horntje (Texel), The Netherlands

${ }^{30}$ Universiteit Utrecht, Faculteit Betawetenschappen, Princetonplein 5, 3584 CC Utrecht, The Netherlands

${ }^{31}$ Universiteit van Amsterdam, Instituut voor Hoge-Energie Fysica, Science Park 105, 1098 XG Amsterdam, The Netherlands

32 Dr. Remeis-Sternwarte and ECAP, Universität Erlangen-Nürnberg, Sternwartstrasse 7, D-96049 Bamberg, Germany

${ }^{33}$ Moscow State University, Skobeltsyn Institute of Nuclear Physics, Leninskie gory, 119991 Moscow, Russia

${ }^{34}$ Dipartimento di Fisica ed Astronomia dell’Università, Viale Andrea Doria 6, I-95125 Catania, Italy

${ }^{35}$ Direction des Sciences de la Matière - Institut de recherche sur les lois fondamentales de l'Univers - Service de Physique des Particules, CEA Saclay, F-91191 Gif-sur-Yvette Cedex, France

${ }^{36}$ IPHC-Institut Pluridisciplinaire Hubert Curien - Université de Strasbourg et CNRS/IN2P3 23 rue du Loess, BP 28, F-67037 Strasbourg Cedex 2, France

${ }^{37}$ ITEP - Institute for Theoretical and Experimental Physics, B. Cheremushkinskaya 25, 117218 Moscow, Russia

${ }^{38}$ Dipartimento di Fisica dell'Università, Via Dodecaneso 33, I-16146 Genova, Italy

${ }^{39}$ University Mohammed I, Laboratory of Physics of Matter and Radiations, B.P.717, Oujda 6000, Morocco Received 2014 February 24; accepted 2014 March 14; published 2014 April 11

\begin{abstract}
A search for cosmic neutrino sources using six years of data collected by the ANTARES neutrino telescope has been performed. Clusters of muon neutrinos over the expected atmospheric background have been looked for. No clear signal has been found. The most signal-like accumulation of events is located at equatorial coordinates
\end{abstract}


R.A. $=-46.8$ and decl. $=-64.9$ and corresponds to a $2.2 \sigma$ background fluctuation. In addition, upper limits on the flux normalization of an $E^{-2}$ muon neutrino energy spectrum have been set for 50 pre-selected astrophysical objects. Finally, motivated by an accumulation of seven events relatively close to the Galactic Center in the recently reported neutrino sample of the IceCube telescope, a search for point sources in a broad region around this accumulation has been carried out. No indication of a neutrino signal has been found in the ANTARES data and upper limits on the flux normalization of an $E^{-2}$ energy spectrum of neutrinos from point sources in that region have been set. The $90 \%$ confidence level upper limits on the muon neutrino flux normalization vary between 3.5 and $5.1 \times 10^{-8} \mathrm{GeV} \mathrm{cm}^{-2} \mathrm{~s}^{-1}$, depending on the exact location of the source.

Key words: astroparticle physics - Galaxy: center - neutrinos

Online-only material: color figures

\section{INTRODUCTION}

The scientific motivation of neutrino telescopes relies on the unique properties of neutrinos, which can be used to observe and study the high-energy universe. Cosmic rays or highenergy photons have intrinsic limitations: the mean free path of gamma-ray photons strongly depends on their energy, while magnetic fields deflect cosmic rays, diluting the information about their origin. Neutrinos are stable, neutral, and weakly interacting particles, and therefore they point directly back to their origin. In addition, neutrinos are expected to originate at the same locations where the acceleration of cosmic rays and the associated production of high-energy photons take place (Halzen \& Hooper 2002; Stecker 2005; Bednarek et al. 2005). The first evidence of such a cosmic neutrino signal has recently been reported by IceCube (Aartsen et al. 2013a, 2013c), including in particular a cluster of events close to the Galactic Center. While this cluster is not statistically significant, González-García et al. (2013) have proposed they may be due to a single point source. The better view of the Southern Hemisphere afforded by the ANTARES neutrino telescope, due to its location in the Mediterranean Sea, provides an increased sensitivity to galactic sources of neutrinos with energies $<100 \mathrm{TeV}$. This is particularly important in order to interpret the cluster of events observed by IceCube close to the Galactic Center.

In this Letter, the results of the search for point sources with the data gathered between 2007 and 2012 with the ANTARES neutrino telescope are presented. After a brief description of the apparatus, the data selection and the corresponding detector performance are presented in Sections 2 and 3, respectively. In Section 4, the search method is explained. The results of the fullsky and candidate sources searches are presented in Section 5. The implications on some recent interpretations of the IceCube results are discussed in Section 6. Finally, the conclusions are given in Section 7.

\section{THE ANTARES NEUTRINO TELESCOPE AND DATA SELECTION}

ANTARES is an underwater neutrino telescope located $40 \mathrm{~km}$ to the South of Toulon (France) in the Mediterranean Sea $\left(42^{\circ} 48^{\prime}\right.$ $\mathrm{N}, 6^{\circ} 10^{\prime} \mathrm{E}$ ) (Ageron et al. 2011). It is made of 12 slender lines spaced by about $65 \mathrm{~m}$, anchored on the seabed at $2475 \mathrm{~m}$ depth and maintained vertical by a buoy. Each line of $350 \mathrm{~m}$ active length comprises 25 floors spaced regularly, each housing three photomultiplier tubes (PMTs) looking downward at an angle of $45^{\circ}$. The detection principle is based on the observation of the Cherenkov light induced by muons produced in charged current interactions of high energy neutrinos inside or near the detector volume. Some of the emitted photons produce a signal in the PMTs ("hits") with the corresponding charge and time information. The hits are used to reconstruct the direction of the muon. In addition, other neutrino signatures such as cascade events are also detected and reconstructed. The current analysis uses muon tracks only, which offer a better angular resolution and larger volume than cascades caused by showering events.

High quality runs are selected from data between 2007 January 29 to 2012 December 31. This measurement period corresponds to a total livetime of 1338 days, which is an increase of $70 \%$ compared to the previous ANTARES pointsource analysis (Adrián-Martínez et al. 2012).

Triggered events are reconstructed using the time and position information of the hits by means of a maximum likelihood (ML) method (Adrián-Martínez et al. 2013). The algorithm consists of a multi-step procedure to fit the direction of the reconstructed muon by maximizing the ML-parameter $\Lambda$, which describes the quality of the reconstruction. In addition, the uncertainty of the track direction angle, $\beta$, is calculated. This calculation is estimated from the uncertainty on the zenith and azimuth angles drawn from the covariance matrix.

Neutrinos and atmospheric muons are simulated with the GENHEN (Brunner 2003) and MUPAGE (Carminati et al. 2008; Bazzotti et al. 2010) packages, respectively. Furthermore, the propagation of the muon tracks is simulated with the KM3 package (Brunner 2003). A data versus simulation comparison of the $\Lambda$ distribution for zenith angles $\theta$ with $\cos \theta<0.1$ can be seen in Figure 1, where the atmospheric neutrino simulation uses the Bartol flux (Agrawal et al. 1996).

Events are selected following a blind procedure on pseudoexperiments before performing the analysis on data. The cuts on reconstructed tracks $\left(\Lambda>-5.2, \beta<1^{\circ}\right.$, and $\left.\cos \theta<0.1\right)$ are chosen so that the neutrino flux needed to make a $5 \sigma$ discovery in $50 \%$ of the experiments is minimized. This selection leads to a final data sample of 5516 events, which includes an estimated $10 \%$ background from mis-reconstructed atmospheric muons.

\section{DETECTOR PERFORMANCE}

For a neutrino energy spectrum proportional to $E^{-2}$, the angular resolution and acceptance for events passing the selection cuts are computed.

An improved modeling of the PMT transit-time distribution compared to Adrián-Martínez et al. (2012) has been used for the simulation. As a result, the estimated median neutrino angular resolution is 0.38 , which corresponds to a $15 \%$ improvement. Figure 2 (left) shows the cumulative distribution of the angle $\Psi$ between the reconstructed muon direction and the true neutrino direction. The distribution is represented both for the whole data set (blue line) and for the previous analysis (dashed red line). 


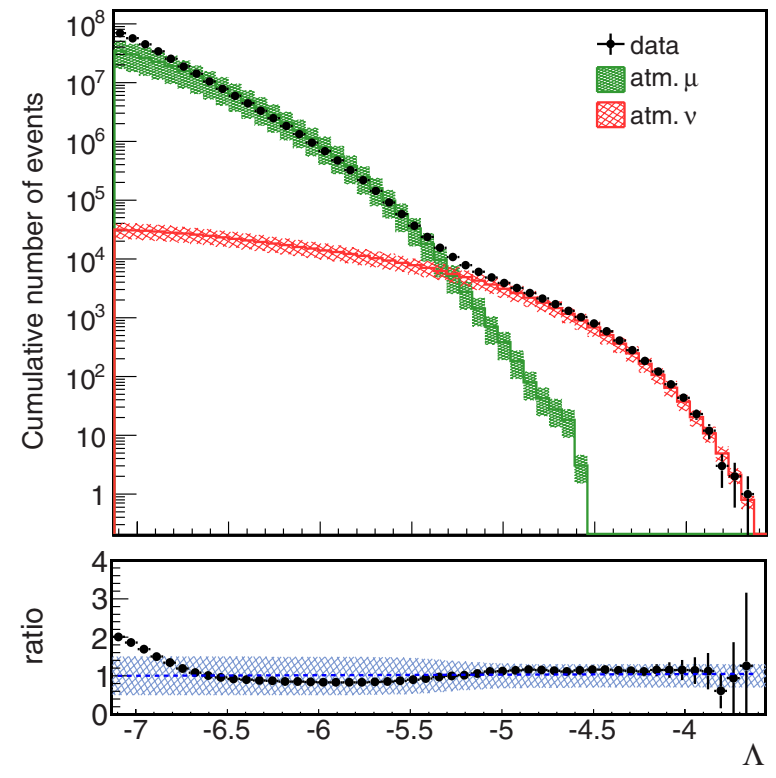

Figure 1. Cumulative distribution of the track reconstruction quality parameter, $\Lambda$, for tracks with $\cos \theta<0.1$ (mostly upgoing) which have an angular error estimate $\beta<1^{\circ}$. Larger values of the $\Lambda$ parameter indicate a better track reconstruction. A better track reconstruction is expected for muon-neutrinos (no upgoing atmospheric muons are expected). The bottom panel shows the ratio between data and simulation. The green (red) distribution corresponds to the simulated atmospheric muons (neutrinos), where a $50 \%(30 \%)$ relative error was assigned (Aguilar et al. 2010; Barr et al. 2006). Data errors correspond to statistical errors only.

(A color version of this figure is available in the online journal.)

The "acceptance" is defined as the quantity that multiplied by a given flux normalization, $\Phi_{0}$, gives the number of signal events. This quantity is proportional to the detector response and depends on the source energy spectrum and declination. The acceptance for a source located at a declination $\delta$ is

$$
A(\delta)=\Phi_{0}^{-1} \int d t \int d E_{v} A_{\mathrm{eff}}\left(E_{v}, \delta\right) \frac{d \Phi}{d E_{v}}
$$

where the time integration extends over the whole period of 1338 days, $A_{\text {eff }}$ is the neutrino effective area, and a source spectrum of $\left(d \Phi / d E_{v}\right)=\Phi_{0} E_{v}^{-2}$ is assumed. The acceptance as a function of the declination $\delta$ is shown in Figure 2 (right).

\section{SEARCH METHOD}

Signal events are expected to accumulate in clusters over a background of diffusely distributed atmospheric neutrinos. The search for clusters is performed using an ML estimation, which describes the data as a mixture of a signal and background probability density functions (PDFs):

$$
\log L_{s+b}=\sum_{i} \log \left[\frac{n_{s}}{N} S_{i}+\left(1-\frac{n_{s}}{N}\right) B_{i}\right] .
$$

Both the background and the signal PDFs, $B_{i}$ and $S_{i}$, respectively, depend on the reconstructed direction, $\boldsymbol{x}_{i}=\left(\alpha_{i}, \delta_{i}\right)$ (where $\alpha_{i}$ and $\delta_{i}$ indicate the reconstructed right ascension and declination, respectively), for the $i$ th event. The parameter $n_{s}$ represents the expected number of signal events for a particular source and $N$, the total number of events in the sample. The signal PDF is defined as

$$
S_{i}=\frac{1}{2 \pi \beta_{i}^{2}} \exp \left(-\frac{\psi_{i}\left(\boldsymbol{x}_{s}\right)^{2}}{2 \beta_{i}^{2}}\right) P_{s}\left(\mathcal{N}_{i}^{\text {hits }}, \beta_{i}\right),
$$

where $\boldsymbol{x}_{s}=\left(\alpha_{s}, \delta_{s}\right)$ indicates the position of the source, $\psi_{i}\left(\boldsymbol{x}_{s}\right)^{2} \approx\left(\alpha_{i}-\alpha_{s}\right)^{2} \cos ^{2}\left(\delta_{s}\right)+\left(\delta_{i}-\delta_{s}\right)^{2}$ indicates the angular distance to the source, and $P_{s}\left(\mathcal{N}_{i}^{\text {hits }}, \beta_{i}\right)$ is the probability for a signal event $i$ at a position $\boldsymbol{x}_{i}$ to be reconstructed with an angular error estimate of $\beta_{i}$ and a number of hits $\mathcal{N}_{i}^{\text {hits }}$. The number of hits $\mathcal{N}_{i}^{\text {hits }}$ is a proxy for the energy of the event.

The background PDF is described as

$$
B_{i}=\frac{B\left(\delta_{i}\right)}{2 \pi} P_{b}\left(\mathcal{N}_{i}^{\text {hits }}, \beta_{i}\right),
$$

where $B\left(\delta_{i}\right)$ is the probability to find an event at a declination $\delta_{i}$ and $P_{b}\left(\mathcal{N}_{i}^{\text {hits }}, \beta_{i}\right)$ is the probability for a background event to be reconstructed with a number of hits $\mathcal{N}_{i}^{\text {hits }}$ and an angular error estimate of $\beta_{i}$.

The significance of any observation is determined by the test statistic, TS, which is defined as TS $=\log L_{s+b}-\log L_{b}$, where $L_{b}$ indicates the likelihood value for the background only case $\left(n_{s}=0\right)$. Larger TS values indicate a lower probability ( $p$-value) of the observation to be produced by the expected background.

\section{FULL-SKY AND CANDIDATE LIST SEARCHES}

A full-sky search and a search on a pre-selected list of candidate sources are performed.
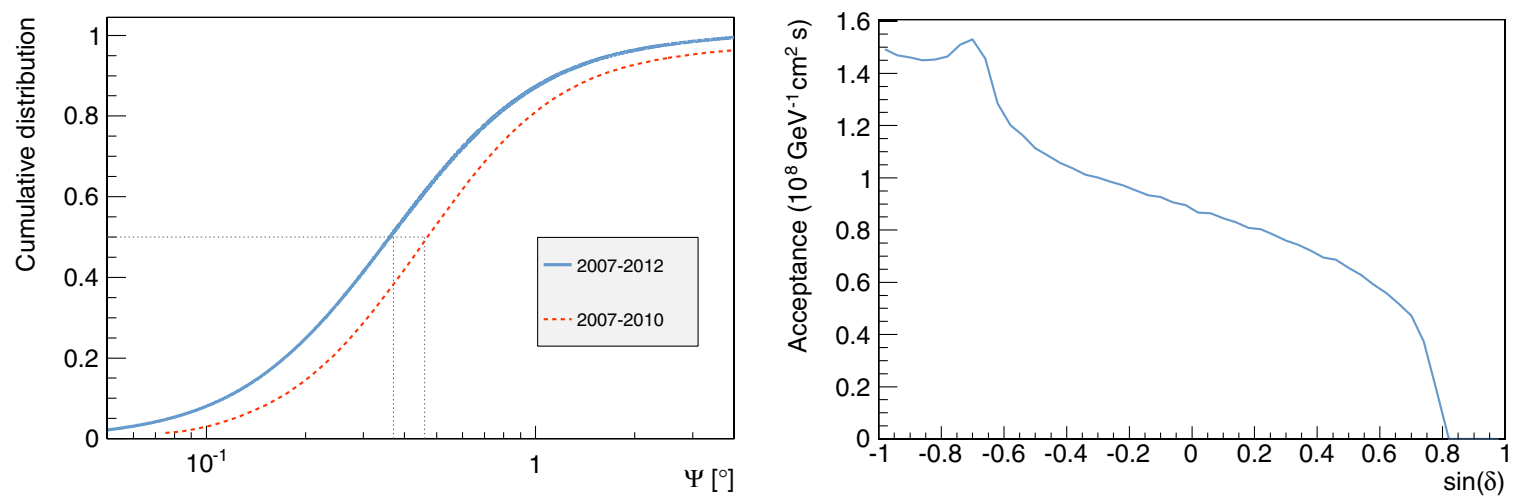

Figure 2. Left: Neutrino angular resolution determined as the median of the cumulative distribution of the reconstruction angle, $\Psi$, for the present data (solid blue line) compared to the 2007-2010 analysis (dashed red line). The black-dotted line indicates the median value. Right: Acceptance (defined in Equation (1)) as a function of the declination $\delta$. An $E^{-2}$ source spectrum has been assumed for both figures.

(A color version of this figure is available in the online journal.) 


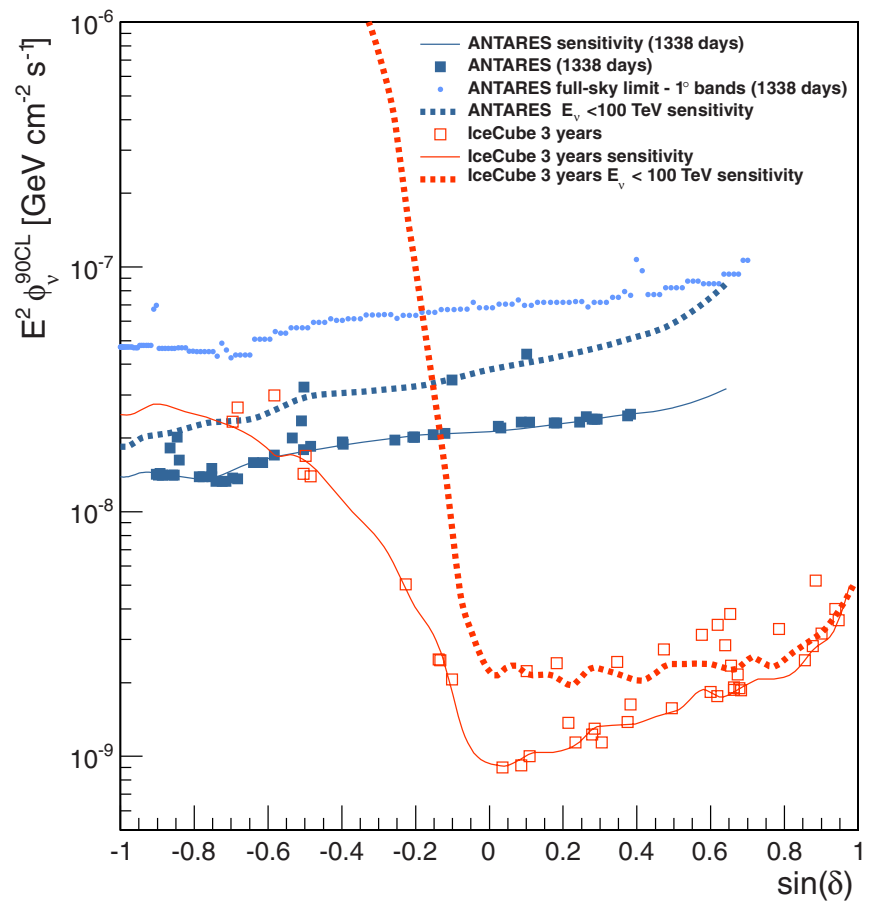

Figure 3. $90 \%$ CL flux upper limits and sensitivities on the muon neutrino flux for six years of ANTARES data. IceCube results are also shown for comparison. The light-blue markers show the upper limit for any point source located in the ANTARES visible sky in declination bands of $1^{\circ}$. The solid blue (red) line indicates the ANTARES (IceCube) sensitivity for a point-source with an $E^{-2}$ spectrum as a function of the declination. The blue (red) squares represent the upper limits for the ANTARES (IceCube) candidate sources. Finally, the dashed dark blue (red) line indicates the ANTARES (IceCube) sensitivity for a point-source and for neutrino energies lower than $100 \mathrm{TeV}$, which shows that the IceCube sensitivity for sources in the Southern hemisphere is mostly due to events of higher energy. The IceCube results were derived from Aartsen et al. (2013b).

(A color version of this figure is available in the online journal.)

The full-sky search looks for an excess of signal events located anywhere in the whole ANTARES visible sky. A preclustering algorithm to select candidate clusters of at least 4 events in a cone of half-opening angle of $3^{\circ}$ is performed. For each cluster, $L_{s+b}$ is maximized by varying the free parameters $\boldsymbol{x}_{s}$ and $n_{s}$. In this analysis, the most significant cluster is found at $(\alpha, \delta)=(-46.8,-64.9)$ with a post-trial $p$-value of $2.7 \%$ (significance of $2.2 \sigma$ using the two-sided convention). This direction is consistent with the most significant cluster found in the previous analysis. The number of fitted signal events is $n_{s}=$ 6.2. A total of $6(14)$ events in a cone of $1^{\circ}\left(3^{\circ}\right)$ around the fitted cluster center are found. Upper limits at the $90 \%$ confidence level (CL) on the muon neutrino flux from point sources located anywhere in the visible ANTARES sky are given by the light blue-dashed line in Figure 3. Each value corresponds to the highest upper-limit obtained in declination bands of $1^{\circ}$.

The second search uses a list of 50 neutrino candidatesource positions at which the likelihood is evaluated. The list of sources with their corresponding pre-trial $p$-values and flux upper limits is presented in Table 1. The largest excess corresponds to HESS J0632+057, with a post-trial $p$-value of $6.1 \%$ ( significance of $1.9 \sigma$ using the two-sided convention). The fitted number of source events is $n_{s}=1.6$. The limits for these 50 selected sources and the overall fixed-source sensitivity of the telescope are reported in Figure 3. The 90\% CL flux upper limits

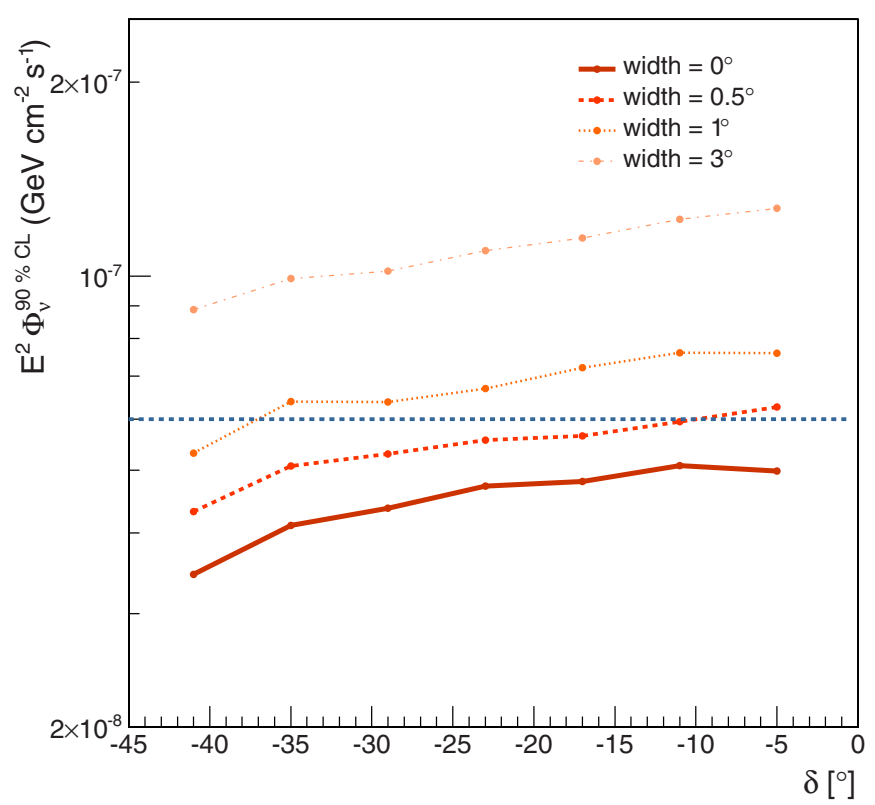

Figure 4. 90\% CL upper limits obtained for different source widths as a function of the declination. The blue horizontal dashed line corresponds to the signal flux given by González-García et al. (2013).

(A color version of this figure is available in the online journal.)

and sensitivities are calculated by using the Neyman method (Neyman 1937).

\section{IMPLICATIONS FOR THE INTERPRETATION OF THE RECENT IceCube RESULTS}

Following the recent evidence of high energy neutrinos by IceCube (Aartsen et al. 2013a), a point source close to the Galactic Center has been proposed to explain the accumulation of seven events in its neighborhood (González-García et al. 2013). The corresponding flux normalization of this hypothetical source $\left(\alpha=-79^{\circ}, \delta=-23^{\circ}\right)$ is expected to be $\Phi_{0}=6 \times 10^{-8} \mathrm{GeV} \mathrm{cm}^{-2} \mathrm{~s}^{-1}$.

This hypothetical source might be located at a different point in the sky due to the large uncertainty of the direction estimates of these IceCube events. The full-sky algorithm with the likelihood presented in Adrián-Martínez et al. (2012) is used, restricted to region of $20^{\circ}$ around the proposed location. The trial factor of this analysis is smaller than in the full-sky search because of the smaller size of the region. In addition to the point source hypothesis, three Gaussian-like source extensions are assumed $\left(0.5,1^{\circ}\right.$ and $\left.3^{\circ}\right)$. As in the full-sky search, a half opening angle of $3^{\circ}$ is used for the pre-clustering selection for source widths smaller than $3^{\circ}$. In the case of the $3^{\circ}$ source assumption, the angle is of $6^{\circ}$.

No significant cluster has been found. Figure 4 shows the $90 \%$ CL flux upper limits obtained for the four assumed different spatial extensions of the neutrino source as a function of the declination. The presence of a point source with a flux normalization of $6 \times 10^{-8} \mathrm{GeV} \mathrm{cm}^{-2} \mathrm{~s}^{-1}$ anywhere in the region is excluded. Therefore, the excess found by IceCube in this region cannot be caused by a single point source. Furthermore, a source width of 0.5 for declinations lower than $-11^{\circ}$ is also excluded. For an $E^{-2}$ spectrum, neutrinos with $E>2 \mathrm{PeV}$ contribute only $7 \%$ to the event rate, hence these results are hardly affected by a cutoff at energies on the order of $\mathrm{PeV}$. 
Table 1

Pre-trial $p$-values, $p$, Fitted Number of Source Events, $n_{s}$, and 90\% CL Flux Limits, $\Phi_{v}^{90 \mathrm{CL}}$, Obtained for the 50 Candidate Sources

\begin{tabular}{|c|c|c|c|c|c|c|c|c|c|c|c|}
\hline Name & $\alpha\left(^{\circ}\right)$ & $\delta\left(^{\circ}\right)$ & $n_{s}$ & $p$ & $\phi_{v}^{90 \mathrm{CL}}$ & Name & $\alpha\left(^{\circ}\right)$ & $\delta\left(^{\circ}\right)$ & $n_{s}$ & $p$ & $\phi_{v}^{90 \mathrm{CL}}$ \\
\hline HESSJ0632+057 & 98.24 & 5.81 & 1.60 & 0.0012 & 4.40 & HESSJ1912+101 & -71.79 & 10.15 & 0.00 & 1.00 & 2.31 \\
\hline HESSJ1741-302 & -94.75 & -30.20 & 0.99 & 0.003 & 3.23 & PKS0426-380 & 67.17 & -37.93 & 0.00 & 1.00 & 1.59 \\
\hline $3 \mathrm{C} 279$ & -165.95 & -5.79 & 1.11 & 0.01 & 3.45 & W28 & -89.57 & -23.34 & 0.00 & 1.00 & 1.89 \\
\hline HESSJ1023-575 & 155.83 & -57.76 & 1.98 & 0.03 & 2.01 & MSH15-52 & -131.47 & -59.16 & 0.00 & 1.00 & 1.41 \\
\hline ESO139-G12 & -95.59 & -59.94 & 0.79 & 0.06 & 1.82 & RGBJ0152+017 & 28.17 & 1.79 & 0.00 & 1.00 & 2.19 \\
\hline CirX-1 & -129.83 & -57.17 & 0.96 & 0.11 & 1.62 & W51C & -69.25 & 14.19 & 0.00 & 1.00 & 2.32 \\
\hline PKS0548-322 & 87.67 & -32.27 & 0.68 & 0.10 & 2.00 & PKS1502+106 & -133.90 & 10.52 & 0.00 & 1.00 & 2.31 \\
\hline GX339-4 & -104.30 & -48.79 & 0.50 & 0.14 & 1.50 & HESSJ1632-478 & -111.96 & -47.82 & 0.00 & 1.00 & 1.33 \\
\hline VERJ0648+152 & 102.20 & 15.27 & 0.59 & 0.11 & 2.45 & HESSJ1356-645 & -151.00 & -64.50 & 0.00 & 1.00 & 1.42 \\
\hline PKS0537-441 & 84.71 & -44.08 & 0.24 & 0.16 & 1.37 & 1ES1101-232 & 165.91 & -23.49 & 0.00 & 1.00 & 1.92 \\
\hline MGROJ1908+06 & -73.01 & 6.27 & 0.21 & 0.14 & 2.32 & HESSJ1507-622 & -133.28 & -62.34 & 0.00 & 1.00 & 1.41 \\
\hline Crab & 83.63 & 22.01 & 0.00 & 1.00 & 2.46 & RXJ0852.0-4622 & 133.00 & -46.37 & 0.00 & 1.00 & 1.33 \\
\hline HESSJ1614-518 & -116.42 & -51.82 & 0.00 & 1.00 & 1.39 & RCW86 & -139.32 & -62.48 & 0.00 & 1.00 & 1.41 \\
\hline HESSJ1837-069 & -80.59 & -6.95 & 0.00 & 1.00 & 2.09 & RXJ1713.7-3946 & -101.75 & -39.75 & 0.00 & 1.00 & 1.59 \\
\hline PKS0235+164 & 39.66 & 16.61 & 0.00 & 1.00 & 2.39 & SS433 & -72.04 & 4.98 & 0.00 & 1.00 & 2.32 \\
\hline Geminga & 98.31 & 17.01 & 0.00 & 1.00 & 2.39 & 1ES0347-121 & 57.35 & -11.99 & 0.00 & 1.00 & 2.01 \\
\hline PKS0727-11 & 112.58 & -11.70 & 0.00 & 1.00 & 2.01 & VelaX & 128.75 & -45.60 & 0.00 & 1.00 & 1.33 \\
\hline PKS2005-489 & -57.63 & -48.82 & 0.00 & 1.00 & 1.39 & HESSJ1303-631 & -164.23 & -63.20 & 0.00 & 1.00 & 1.43 \\
\hline PSRB1259-63 & -164.30 & -63.83 & 0.00 & 1.00 & 1.41 & LS5039 & -83.44 & -14.83 & 0.00 & 1.00 & 1.96 \\
\hline HESSJ1503-582 & -133.54 & -58.74 & 0.00 & 1.00 & 1.41 & PKS2155-304 & -30.28 & -30.22 & 0.00 & 1.00 & 1.79 \\
\hline PKS0454-234 & 74.27 & -23.43 & 0.00 & 1.00 & 1.92 & Galactic Center & -93.58 & -29.01 & 0.00 & 1.00 & 1.85 \\
\hline PKS1454-354 & -135.64 & -35.67 & 0.00 & 1.00 & 1.70 & CentaurusA & -158.64 & -43.02 & 0.00 & 1.00 & 1.36 \\
\hline HESSJ1834-087 & -81.31 & -8.76 & 0.00 & 1.00 & 2.06 & W44 & -75.96 & 1.38 & 0.00 & 1.00 & 2.23 \\
\hline HESSJ1616-508 & -116.03 & -50.97 & 0.00 & 1.00 & 1.39 & IC443 & 94.21 & 22.51 & 0.00 & 1.00 & 2.50 \\
\hline H2356-309 & -0.22 & -30.63 & 0.00 & 1.00 & 2.35 & $3 \mathrm{C} 454.3$ & -16.50 & 16.15 & 0.00 & 1.00 & 2.39 \\
\hline
\end{tabular}

Note. The fluxes are in units of $10^{-8} \mathrm{GeV} \mathrm{cm}^{-2} \mathrm{~s}^{-1}$.

\section{CONCLUSION}

In this Letter, the results of a search for neutrino point sources with six years of ANTARES data (2007-2012) are presented using two complementary analyses: first, a scan for point sources of the ANTARES visible sky; second, a search for correlations of events with a pre-selected list of candidate sources for neutrino emission. In the first case, the most significant cluster has a post-trial $p$-value of $2.7 \%$ (a significance of $2.2 \sigma$ ). In the case of the candidate list study, the largest excess corresponds to HESS J0632+057 with a posttrial $p$-value of $6.1 \%(1.9 \sigma)$. Both results are compatible with a pure background hypothesis. The derived flux upper limits are the most restrictive in a significant part of the Southern sky. The possibility that the accumulation of seven events reported by IceCube near the Galactic Center is produced by a single point source has been excluded. These results show the potential of neutrino telescopes in the Northern hemisphere, such as the planned KM3NeT observatory (Leisos et al. 2013), to interpret the increasing evidence of cosmic neutrino fluxes.

The authors acknowledge the financial support of the funding agencies: Centre National de la Recherche Scientifique (CNRS), Commissariat à l'Énegie Atomique et aux Énergies Alternatives (CEA), Commission Européenne (FEDER fund and Marie Curie Program), Région Alsace (contrat CPER), Région Provence-Alpes-Côte d'Azur, Département du Var and Ville de La Seyne-sur-Mer, France; Bundesministerium für Bildung und Forschung (BMBF), Germany; Istituto Nazionale di Fisica Nucleare (INFN), Italy; Stichting voor Fundamenteel Onderzoek der Materie (FOM), Nederlandse organisatie voor Wetenschappelijk Onderzoek (NWO), the Netherlands; Council of the President of the Russian Federation for young scientists and leading scientific schools supporting grants, Russia; National Authority for Scientific Research (ANCS), Romania; Ministerio de Ciencia e Innovación (MICINN), Prometeo of Generalitat Valenciana and MultiDark, Spain; Agence de l'Oriental and CNRST, Morocco. We also acknowledge the technical support of Ifremer, AIM, and Foselev Marine for the sea operation and the CC-IN2P3 for the computing facilities.

\section{REFERENCES}

Aartsen, M. G., Abbasi, R., Abdou, Y., et al. (IceCube Collaboration) 2013a, PhRvL, 111, 021103

Aartsen, M. G., Abbasi, R., Abdou, Y., et al. (IceCube Collaboration) 2013b, ApJ, 779, 132

Aartsen, M. G., Abbasi, R., Abdou, Y., et al. (IceCube Collaboration) 2013c, Sci, 342, 1242856

Adrián-Martínez, S., Samarai, I. Al, Albert, A., et al. (ANTARES Collaboration) 2012, ApJ, 760, 53

Adrián-Martínez, S., Samarai, I. Al, Albert, A., et al. (ANTARES Collaboration) 2013, JCAP, 03, 006

Ageron, M., Aguilar, J. A., Samarai, I. Al, et al. (ANTARES Collaboration) 2011, NIMPA, 656, 11

Agrawal, V., Gaisser, T. K., Lipari, P., \& Stanev, T. 1996, PhRvD, 53, 1314

Aguilar, J. A., Albert, A., Anton, G., et al. (ANTARES Collaboration) 2010, APh, 34, 179

Barr, G. D., Gaisser, T. K., Robbins, S., \& Stanev, T. 2006, PhRvD, 74, 094009

Bazzotti, M., Carminati, G., Margiotta, A., \& Spurio, M. 2010, CoPhC, 181,835

Bednarek, W., Burgio, G. F., \& Montaruli, T. 2005, NewAR, 49, 1

Brunner, J. 2003, in VLVnT Workshop (Amsterdam), ANTARES Simulation Tools, ed. E. de Wolf (Amsterdam: NIKHEF), 109 http://www.vlvnt.nl/ proceedings.pdf

Carminati, G., Bazzotti, M., Margiotta, A., \& Spurio, M. 2008, CoPhC, 179,915

González-García, M. C., Halzen, F., \& Niro, V. 2013, arXiv:1310.7194

Halzen, F., \& Hooper, D. 2002, RPPh, 65, 1025

Leisos, A., Tsirigotis, A. G., Tzamarias, S. E., et al. (KM3NeT Consortium) 2013, NIMPA, 725, 55

Neyman, J. 1937, RSPTA, 236, 333

Stecker, F. W. 2005, PhRvD, 72, 107301 